\title{
Polimorfismos genéticos de APOA5 se asocian a hipertri- gliceridemia e hiperglicemia en individuos chilenos con enfermedad coronaria y controles
}

\author{
Nicolás Saavedra ${ }^{1,2}$, Alejandro Cuevas ${ }^{1,2}$, Alfonso Hernández ${ }^{1,2}$, José Caamaño ${ }^{1,2}$, Priscilla \\ Jaramillo ${ }^{1,2}$, Fernando Lanas ${ }^{2,3}$, Luis A. Salazar ${ }^{1,2}$ \\ ${ }^{1}$ Laboratorio de Biología Molecular y Farmacogenética, Departamento de Ciencias Básicas; \\ ${ }^{2}$ Núcleo de Desarrollo Científico - Tecnológico en Biorecursos (BIOREN), ${ }^{3}$ Departamento de \\ Medicina Interna, Facultad de Medicina, Universidad de La Frontera, Temuco, Chile. \\ Trabajo financiado por FDI-MINEDUC \& DIUFRO DI09-2012, Dirección de Investigación y \\ Desarrollo, Universidad de La Frontera.
}

Recibido el 19 de Noviembre de 2008, Aceptado el 1 de Marzo de 2010

Rev Chil Cardiol 2010; 29: 19-27

\section{Resumen}

Introducción: Diversas variantes genéticas han sido relacionadas al desarrollo de enfermedad coronaria y/o sus factores de riesgo; entre ellas, los polimorfismos S19W y -1131T>C del gen que codifica para la apolipoproteína A5 (APOA5). Así, el objetivo del presente estudio fue investigar la posible asociación entre las variantes S19W y $-1131 T>C$ del gen APOA5 y enfermedad coronaria en individuos chilenos.

Métodos: Se evaluaron 425 sujetos adultos, no relacionados; 209 pacientes con enfermedad coronaria (EC) comprobada por angiografía (estenosis > 70\%), con edades entre 33 y 74 años, y 216 individuos controles (30 a 68 años). La genotipificación de los polimorfismos S19W y -1131T>C del gen APOA5 fue realizada mediante la técnica de PCR-RFLP.

Resultados: La distribución de los genotipos para el polimorfismo S19W del gen APOA5 en el grupo casos (SS: 80\%, SW: $19 \%$ y WW: 1\%) y en el grupo control (SS: $82 \%$, SW: $17 \%$ y WW: $1 \%$ ) fue semejante (p=NS). La distribución genotípica para el polimorfismo -1131T>C en pacientes con EC (TT: 56\%, TC: 37\%, y CC: $7 \%$ ) y controles (TT: 63\%, TC: $30 \%$ y CC: $7 \%$ ) fue similar ( $p=N S)$. Las ORs relacionadas a los alelos mutados $19 W(1.12$; I.C.95\%, $0.72-1.74, p=N S)$ y -1131 C (1.19; I.C.95\%, $0.87-1.63, p=N S)$, confirman la ausencia de asociación. Por otro lado, las concentraciones de triglicéridos y glucosa en ayunas fueron significativamente más elevadas en los sujetos portadores de los alelos $19 W$ y -1131C, tanto en casos como en controles $(p<0.05)$.

Conclusión: La asociación observada entre las variantes genéticas de APOA5 y las altas concentraciones séricas de triglicéridos y glucosa, en ambos grupos, sugiere que estos polimorfismos podrían contribuir al desarrollo de la dislipidemia diabética; un reconocido factor de riesgo para enfermedad arterial coronaria.

Correspondencia: Dr. Luis Antonio Salazar

Departamento de Ciencias Básicas

Facultad de Medicina, Universidad de La Frontera

Av. Francisco Salazar 01145

Casilla 54-D, Temuco. Fono: 45-592895 - Fax: 45 - 592832

Correo Electrónico: Isalazar@ufro.cl 


\section{Genetic polymorphisms of APOA5 are associated with hypertriglyceridemia and hyperglycemia in Chilean subjects with coronary artery disease and controls}

Background: Several genetic variants have been linked to the development of coronary heart disease and / or their risk factors, including the S19W and-1131T>C polymorphisms of the gene that encodes apolipoprotein A5 (APOA5). Thus, the objective of this study was to investigate the possible association between S19W and -1131 T $>C$ genetic variants of APOA5 and coronary disease in Chilean individuals.

Methods: We evaluated 425 not related subjects; 209 patients with coronary artery disease (CAD) confirmed by angiography (stenosis > 70\%), aged between 33 and 74 years, and 216 control individuals (30 to 68 years). The genotyping of S19W and -1131T>C polymorphisms of APOA5 gene was evaluated by PCR-RFLP.

Results: The genotype distribution of S19W polymorphism of APOA5 gene in CAD patients (SS: 80\%, SW: $19 \%$ WW: $1 \%$ ) and controls (SS: $82 \%$, SW: $17 \%$ WW: $1 \%$ ) was similar ( $p=N S)$. In the same way, the genotype distribution of -1131 T>C genetic variant in CAD subjects (TT: $56 \%$, TC: $37 \%$, and CC: $7 \%$ ) and controls (TT: $63 \%$, TC: $30 \%$ and CC: $7 \%)$ was equivalent $(p=N S)$. The Odds ratios related to the mutant alleles $19 \mathrm{~W}(1.12,95 \% \mathrm{Cl}, 0.72-1.74$, $p=N S)$ and $-1131 \mathrm{C}(1.19,95 \% \mathrm{Cl}, 0.87-1.63, p=N S)$ confirms the absence of association. On the other hand, the triglycerides and fasting glucose concentrations were significantly higher in subjects carrying the alleles $19 \mathrm{~W}$ and -1131C, in both groups, CAD patients and controls $(p<0.05)$.

Conclusion: The observed association between genetic variants of APOA5 and higher serum levels of triglycerides and glucose, in both groups, suggesting that these polymorphisms could be contribute to the development of diabetic dyslipidemia, a known risk factor for coronary artery disease.

Key words: Coronary artery disease; APOA5 gene; Cardiovascular risk factors

\section{Introducción}

Las enfermedades cardiovasculares (ECV) se han convertido en un importante problema de salud pública en las últimas décadas, siendo responsables, en el año 2000 , del $31 \%$ de la totalidad de las muertes en Latinoamérica ${ }^{1}$. En nuestro país, la mortalidad asociada a ECV es del orden de un $28 \%$, siendo la principal causa de muerte. Además, un $55 \%$ de la población tiene riesgo de padecer una patología de este tipo, de acuerdo con la encuesta nacional de salud del año 2003 (Ministerio de Salud).

Diversos factores han sido relacionados al origen de la enfermedad coronaria (EC), entre éstos la hipertrigliceridemia. Estudios epidemiológicos muestran que los triglicéridos (TG) aumentados son un factor de riesgo independiente de $\mathrm{EC}^{2,3}$. Debido a la complejidad de la hipertrigliceridemia, existen varios orígenes posibles para este desorden, incluyendo causas secundarias como el alcoholismo, la diabetes mellitus y la obesidad. Actualmente, con la ayuda de técnicas moleculares se ha observado que los factores genéticos tienen un papel importante en el metabolismo y regulación de los triglicéridos. Así, diversos genes candidatos han sido investigados, entre ellos el gen que codifica para la apolipoproteína A-V (APO AV).

La apo A-V fue identificada en el año 2001, simultáneamente por dos grupos de investigadores ${ }^{4,5}$. Se compone de 366 aminoácidos, se produce en el hígado y es codificada por el gen APOA5 que se encuentra íntimamente asociado al locus AI/CIII/AIV, en el cromosoma 11q23. Esta apoliproteína está fuertemente asociada al metabolismo de los triglicéridos, pues se ha detectado en VLDL, HDL y quilomicrones ${ }^{5,6}$. Es importante destacar que la alteración de la concentración de estas lipoproteínas también se relaciona con EC. 
Estudios en ratones transgénicos revelaron que una sobreexpresión de apo A-V humana causa una disminución de los niveles de triglicéridos y HDL-colesterol, mientras que en ratones knockout para este gen, los TG aumentan ${ }^{7}$, dejando en evidencia el importante rol de la apo A-V en el metabolismo lipídico. Se cree que el mecanismo por el cual interviene, es mediado por un aumento de la actividad de la lipoproteínlipasa $(\mathrm{LPL})^{8}$ o mediante interacción con proteoglicanos que atan LPL en la superficie celular ${ }^{9}$, aumentando la lipólisis de las VLDL y disminuyendo así, la concentración de TG en ellas (Figura 1). También se ha sugerido que apo A-V podría aumentar el aclaramiento de las VLDL, posiblemente asociado a una disminución de apo B y apo CIII, y de esta forma disminuir el nivel postprandial de triglicéridos ${ }^{10}$.

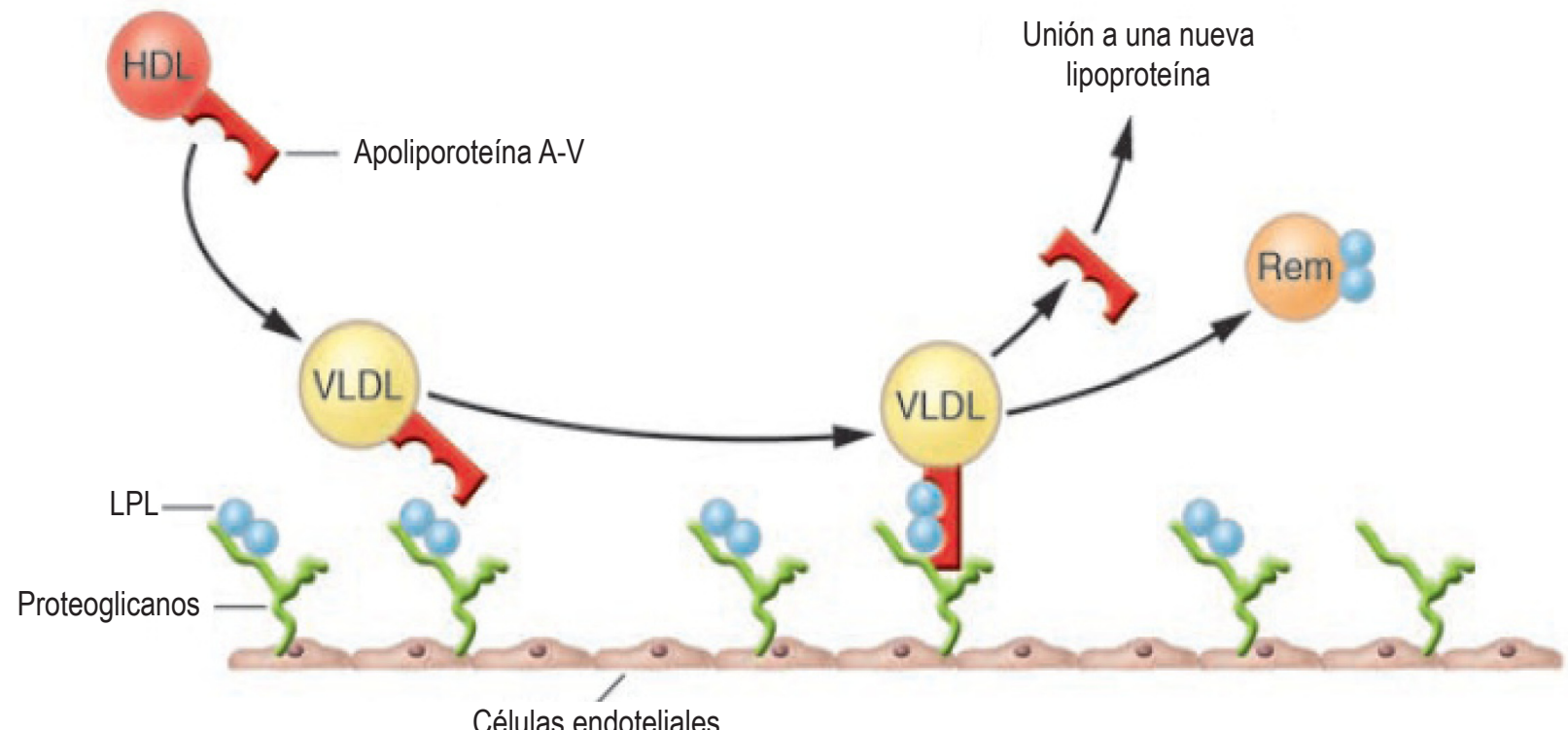

Figura1: Disminución de triglicéridos mediada por la apolipoproteína A-V. Adaptado de Merkel \& Heeren.

Diversas variantes del gen $A P O A 5$ han sido asociadas al aumento de los niveles séricos de triglicéridos en diferentes poblaciones. Entre éstas, las dos más estudiadas son: -1131T>C (región promotora) y c.56 $\mathrm{G}>\mathrm{C}(\mathrm{S} 19 \mathrm{~W}){ }^{11-14}$. La presencia de estos polimorfismos se han asociado a una disminución de la expresión del gen $A P O A 5$, lo que condicionaría un aumento en los niveles de triglicéridos ${ }^{15,16}$, y a una mayor predisposición a padecer diabetes mellitus tipo $2^{17}$; ambos factores de riesgo reconocidos de enfermedad coronaria.

Varios estudios realizados en poblaciones de origen oriental y europea, han demostrado una asociación importante entre EC y los polimorfismos mencionados ${ }^{18-21}$.

Sin embargo, esta asociación no ha sido confirmada por todos los autores ${ }^{22}$, existiendo aún discrepancias al respecto.

Así, el objetivo del presente trabajo fue investigar la posible asociación entre las variantes S19W y -1131T>C del gen APOA5 y enfermedad coronaria en individuos chilenos.

\section{Material y métodos}

\section{Sujetos de estudio}

El presente estudio analizó un total de 425 sujetos 
adultos no relacionados; 209 individuos con diagnóstico de enfermedad arterial coronaria (historia y ECG de infarto al miocardio o enfermedad coronaria comprobada por angiografía con estenosis superior al 70\%), provenientes del Hospital Hernán Henríquez Aravena de la ciudad de Temuco. Por su parte, el grupo control quedó integrado por 216 individuos sin enfermedad coronaria, verificada por la ausencia de angina y electrocardiograma sin evidencias de isquemia o infarto; todos provenientes de la IX Región de La Araucanía.

En este estudio, hipertensión arterial fue definida como los valores medidos de presión arterial sistólica $\geq 140 \mathrm{~mm}$ Hg y/o presión arterial diastólica $\geq 90 \mathrm{~mm} \mathrm{Hg}$ o en uso de tratamiento antihipertensivo. Diabetes mellitus fue definida tras la medición de glucosa en ayunas y obtención de valores $\geq 126 \mathrm{mg} / \mathrm{dl}(7 \mathrm{mmol} / \mathrm{l})$ o en uso de tratamiento hipoglicemiante. Hipercolesterolemia fue definida por valores de colesterol total $\geq 240 \mathrm{mg} / \mathrm{dl}$ en ayunas. La presencia de tabaquismo fue definida como ex - fumadores y fumadores habituales. Mediante encuesta escrita, se obtuvieron también datos de sus hábitos alimentarios, consumo de bebidas alcohólicas e historia familiar de enfermedad cardiovascular precoz (parientes de primer grado $<55$ años para hombres $y<65$ años para mujeres).

Todos los individuos incluidos en este estudio aprobaron su participación mediante la firma de un consentimiento informado, el cual fue previamente aprobado por el Comité de Ética Científica del Servicio de Salud Araucanía Sur (Temuco, IX Región de La Araucanía).

\section{Determinaciones bioquímicas}

Para las determinaciones de laboratorio se obtuvieron muestras de sangre venosa sin anticoagulante, previo ayuno de 12 horas. La concentración de colesterol total y triglicéridos fue determinada por métodos enzimático-colorimétricos ${ }^{23,24}$; el colesterol HDL-C se midió previa precipitación selectiva de las LDL y VLDL con ácido fosfotúngstico e iones magnesio y posterior determinación por el método CHOD-PAP' la concentración de colesterol de las LDL (LDL- C) fue calculada mediante la formula de Friedewald ${ }^{26}$.

Las concentraciones séricas de glucosa y ácido úrico fueron determinadas mediante métodos enzimático-colorimétricos ${ }^{27,28}$.

\section{Genotipificación de las variantes $-1131 \mathrm{~T}>\mathrm{C}$ y $\mathrm{S} 19 \mathrm{~W}$ del gen APOA5}

El ADN genómico fue extraído a partir de sangre total anticoagulada con EDTA mediante la técnica de precipitación salina descrita por Salazar et al.29. Para el estudio de las variantes $-1131 \mathrm{~T}>\mathrm{C}$ y $\mathrm{S} 19 \mathrm{~W}$ se amplificaron fragmentos de 154 y 157 pares de bases (bp), respectivamente, mediante la reacción en cadena de la polimerasa (PCR) en un termociclador MyCycler (Bio-Rad, California, EE.UU.) usando partidores previamente descritos ${ }^{30}$.

El protocolo de PCR utilizado fue: $200 \mathrm{nM}$ de cada primer, dNTPs $0,2 \mathrm{nM}$, cloruro de magnesio $\left(\mathrm{MgCl}_{2}\right)$ $2 \mathrm{mM}, 1 \mathrm{U}$ de Taq Polimerasa (Fermentas, Lituania), buffer 1X, $200 \mathrm{ng}$ de ADN y agua destilada estéril, para un volumen final de $50 \mu \mathrm{L}$. La mezcla de reacción se sometió a una denaturación inicial a $98^{\circ} \mathrm{C}$ durante 3 minutos y 30 ciclos compuestos por denaturación de 1 minuto a $95^{\circ} \mathrm{C}$, hibridación por 1 minuto a $58^{\circ} \mathrm{C}$ $(-1131 \mathrm{~T}>\mathrm{C}) \circ 62^{\circ} \mathrm{C}(\mathrm{S} 19 \mathrm{~W})$, y una fase de extensión de 1 minuto a $72^{\circ} \mathrm{C}$, seguidos de una extensión final de 10 minutos a $72^{\circ} \mathrm{C}$. El producto amplificado se evaluó en gel de agarosa al $2 \%$ teñido con bromuro de etidio $(0.5 \mathrm{mg} / \mathrm{L})$ y visualizado en un sistema de fotodocumentación digital, E-Box 1000 (Vilber Lourmat, Francia).

Los productos de amplificación fueron sometidos a digestión enzimática utilizando las endonucleasas FastDigest Taql para S19W y Tru1l para $-1131 \mathrm{~T}>\mathrm{C}$ (Fermentas, Lituania), obteniendo para el alelo $\mathrm{T}$ de la variante -1131 , dos fragmentos (133 y $21 \mathrm{bp})$ y un único fragmento (154bp) para el alelo $\mathrm{C}$. En cuanto a la variante S19W, se obtuvo dos fragmentos para el alelo $S$ (134 y 23bp) y un único fragmento para el alelo $W$ (157bp). Los productos de restricción fueron evaluados 
Polimorfismos genéticos de APOA5 se asocian a hipertrigliceridemia e hiperglicemia en individuos chilenos con enfermedad...

mediante electroforesis en gel de agarosa al $3 \%$.

\section{Control de calidad de las determinaciones bioquímicas y moleculares}

La exactitud de las determinaciones bioquímicas fue controlada mediante la utilización de sueros comerciales normales y patológicos (Human, Alemania). La posibilidad de contaminación en los análisis moleculares fue excluida por la utilización de controles de reactivos en cada serie de amplificación. La correcta genotipificación de los polimorfismos del gen APOA5 fue confirmada mediante la repetición al azar del $10 \%$ de los análisis previamente realizados. La concordancia encontrada fue de $100 \%$.

\section{Análisis estadístico}

El análisis de los datos obtenidos se realizó utilizando el programa SigmaStat para Windows, versión 2.0 (San Rafael, CA, EE.UU). Todas las variables continuas se expresan como media \pm desviación estándar. La asociación entre las variables analizadas fue comprobada mediante el test $t$ de Student. Además, se utilizó el test de Chi - cuadrado $\left(\chi^{2}\right)$ para el análisis de las variables no continuas y para verificar el equilibrio Hardy - Weinberg. Las Odds ratios (ORs) y sus respectivos intervalos de confianza (I.C) de 95\%, asociadas a los alelos mutados (19W y -1131C) fueron también calculadas. El nivel de significancia estadística considerado en este estudio fue $p<0.05$.

\section{Resultados}

\section{Características de los sujetos}

Las características clínicas, demográficas y de laboratorio de los individuos evaluados en este estudio se resumen en la Tabla 1. Como puede ser comprobado, se encontraron diferencias significativas entre los grupos investigados en relación a las concentraciones séricas de colesterol total, LDL-C, HDL-C, triglicéridos, ácido úrico y glucosa $(p<0.001)$. Entre los parámetros clínicos, se observó que los individuos con EC presentaron valores de presión arterial sistólica y diastólica mayores que el grupo control $(p<0.05)$.

Tabla 1. Características clínicas y demográficas de los individuos con enfermedad coronaria (casos) y controles.

\begin{tabular}{|c|c|c|c|}
\hline Variables & $\begin{array}{l}\text { Casos } \\
\text { (209) }\end{array}$ & $\begin{array}{c}\text { Controles } \\
(216)\end{array}$ & $p^{*}$ \\
\hline Edad, años & $62 \pm 10$ & $42 \pm 8$ & $<0.001$ \\
\hline Hombres, \% & 64 & 56 & 0.109 \\
\hline Diabetes, \% & 32 & 5 & $<0.001$ \\
\hline PAS, mm Hg & $140 \pm 26$ & $125 \pm 23$ & $<0.001$ \\
\hline PAD, $\mathrm{mm} \mathrm{Hg}$ & $80 \pm 18$ & $75 \pm 9$ & 0.020 \\
\hline $\mathrm{IMC}, \mathrm{kg} / \mathrm{m}^{2}$ & $28.2 \pm 4.5$ & $25.8 \pm 4.5$ & $<0.001$ \\
\hline Fumadores, \% & 64 & 41 & $<0.001$ \\
\hline Hipercolesterolemia, \% & 80 & 20 & $<0.001$ \\
\hline Hipertensión, \% & 79 & 26 & $<0.001$ \\
\hline Angina, \% & 77 & 0 & $<0.001$ \\
\hline IAM, \% & 66 & 0 & $<0.001$ \\
\hline Historia familiar de EC, \% & 32 & 0 & $<0.001$ \\
\hline \multicolumn{4}{|l|}{ Número de vasos obstruidos } \\
\hline 1 & $28 \%$ & 0 & --- \\
\hline 2 & $24 \%$ & 0 & -- \\
\hline 3 & $48 \%$ & 0 & --- \\
\hline Colesterol total, $\mathrm{mg} / \mathrm{dL}$ & $214 \pm 51$ & $175 \pm 33$ & $<0.001$ \\
\hline $\mathrm{LDL}-\mathrm{C}, \mathrm{mg} / \mathrm{dL}$ & $127 \pm 42$ & $95 \pm 27$ & $<0.001$ \\
\hline $\mathrm{HDL}-\mathrm{C}, \mathrm{mg} / \mathrm{dL}$ & $32 \pm 8$ & $49 \pm 12$ & $<0.001$ \\
\hline Triglicéridos, $\mathrm{mg} / \mathrm{dL}$ & $182 \pm 193$ & $114 \pm 86$ & $<0.001$ \\
\hline Glucosa, mg/dL & $113 \pm 42$ & $4.5 \pm 1.5$ & $<0.001$ \\
\hline Ácido úrico, $\mathrm{mg} / \mathrm{dL}$ & $5.6 \pm 1.6$ & $4.5 \pm 1.5$ & $<0.001$ \\
\hline
\end{tabular}

EC: Enfermedad coronaria, HDL-C: Colesterol de las HDL, LDL-C: colesterol de las LDL, PAS Presión arterial sistólica, PAD: Presión arterial sistólica, PAD: Presión arterial diastólica. *Test $t$ de Student o Chi-cuadrado. 
Además, la presencia de diabetes mellitus tipo 2, hipercolesterolemia, consumo de tabaco, hipertensión arterial, IAM e historia familiar de EC fue significativamente mayor en los pacientes con EC $(p<0,001)$.

\section{Análisis de los polimorfismos del gen APOA5}

La distribución genotípica y la frecuencia relativa de los alelos para los polimorfismos del gen APOA5 son mostradas en la Tabla 2.

La distribución de los genotipos para el polimorfismo S19W en el grupo casos (SS: $80 \%$, SW: $19 \%$ y WW: $1 \%$ ) y en el grupo control (SS: $82 \%$, SW: $17 \%$ y WW: $1 \%$ ) fue semejante $(p=N S)$. La distribución genotípica para el polimorfismo -1131T >C en pacientes con EC (TT: $56 \%$, TC: $37 \%$, y CC: $7 \%$ ) y controles (TT: $63 \%$, TC: $30 \%$ y CC: $7 \%$ ) fue similar ( $p=N S$ ). Las ORs relacionadas a los alelos mutados $19 \mathrm{~W}(1.12$; I.C.95\%, $0.72-1.74, p=N S)$ y -1131 C (1.19; I.C.95\%, $0.87-1.63, p=N S)$, confirman la ausencia de asociación.

Por otro lado, las concentraciones de triglicéridos y glucosa en ayunas fueron significativamente más elevadas en los sujetos portadores de los alelos $19 \mathrm{~W}$ y $-1131 \mathrm{C}$, tanto en casos como en controles $(p<0.05$, Tablas 3 y 4 ).

Tabla 2. Distribución de genotipos y frecuencia relativa de alelos para los polimorfismos $\mathrm{S} 19 \mathrm{~W}$ y $-113 \mathrm{~T}>\mathrm{C}$ del gen $A P O A 5$ en individuos con enfermedad arterial coronaria (casos) y controles.

\begin{tabular}{lcccc}
\hline Variables & Genotipos & $\begin{array}{c}\text { Casos } \\
(209)\end{array}$ & $\begin{array}{c}\text { Controles } \\
(216)\end{array}$ & $p^{*}$ \\
\hline S19W & SS & $80 \%(167)$ & $82 \%(177)$ & $0.680^{*}$ \\
& SW & $19 \%(39)$ & $17 \%(37)$ & \\
WW & $1 \%(3)$ & & \\
& Alelos & & & 0.698 \\
-1131T>C & S & 0.89 & 0.90 & 0.313 \\
& W & 0.11 & $63 \%(136)$ & \\
& TT & $56 \%(117)$ & $70 \%(65)$ & 0.320 \\
& TC & $37 \%(77)$ & & \\
& CC & $7 \%(15)$ & 0.78 & 0.22 \\
\end{tabular}

Número de individuos en paréntesis. *Test de Chi-cuadrado. "Modelo Aditivo.

Equilibrio de Hardy-Weinberg:

S19W : Casos $\chi^{2}=0.170, p=0.677$; Controles $\chi^{2}=0.002, p=0.966$

$-113 T>C$ : Casos $\chi^{2}=0.225, p=0.630$; Controles $\chi^{2}=3.263, p=0.0 .71$

Tabla 3. Concentraciones séricas de lípidos y glucosa (media $\pm \mathrm{DE}$ ) de pacientes con enfermedad coronaria y controles, de acuerdo a los diferentes genotipos del polimorfismo S19W del gen APOA5.

\begin{tabular}{|c|c|c|c|c|c|c|}
\hline & \multicolumn{3}{|c|}{ Casos } & \multicolumn{3}{|c|}{ Controles } \\
\hline & $\begin{array}{c}\text { SS } \\
(167)\end{array}$ & $\begin{array}{c}S W+W W \\
(42)\end{array}$ & $P^{*}$ & $\begin{array}{c}\text { SS } \\
(177)\end{array}$ & $\begin{array}{c}S W+W W \\
\text { (39) }\end{array}$ & $P^{*}$ \\
\hline Glucosa (mg/mL) & $104 \pm 30$ & $127 \pm 49$ & 0.009 & $86 \pm 10$ & $112 \pm 63$ & 0.001 \\
\hline Colesterol (mg/mL) & $203 \pm 55$ & $196 \pm 51$ & 0.609 & $174 \pm 31$ & $170 \pm 27$ & 0.569 \\
\hline HDL- colesterol (mg/mL) & $32 \pm 7$ & $32 \pm 9$ & 0.715 & $52 \pm 11$ & $50 \pm 12$ & 0.349 \\
\hline LDL- colesterol (mg/mL) & $117 \pm 39$ & $113 \pm 32$ & 0.640 & $102 \pm 29$ & $100 \pm 32$ & 0.810 \\
\hline Triglicéridos (mg/mL) & $145 \pm 81$ & $316 \pm 363$ & $<0.001$ & $128 \pm 71$ & $189 \pm 152$ & 0.012 \\
\hline
\end{tabular}

Número de individuos entre paréntesis. * Test "t" de Student. 
Polimorfismos genéticos de APOA5 se asocian a hipertrigliceridemia e hiperglicemia en individuos chilenos con enfermedad...

Tabla 4. Concentraciones séricas de lípidos y glucosa (media $\pm \mathrm{DE}$ ) de pacientes con enfernedad coronaria y controles, de acuerdo a los diferentes genotipos del polimorfismo -1131T>C del gen APOA5.

\begin{tabular}{|c|c|c|c|c|c|c|c|c|}
\hline & \multicolumn{4}{|c|}{ Casos } & \multicolumn{4}{|c|}{ Controles } \\
\hline & $\begin{array}{c}\text { TT } \\
(117)\end{array}$ & $\begin{array}{c}\mathrm{TC} \\
(77)\end{array}$ & $\begin{array}{l}\text { CC } \\
(15)\end{array}$ & $p^{*}$ & $\begin{array}{c}\text { TT } \\
(136)\end{array}$ & $\begin{array}{c}\mathrm{TC} \\
(65)\end{array}$ & $\begin{array}{c}\text { CC } \\
(15)\end{array}$ & $p^{*}$ \\
\hline Glucosa (mg/mL) & $106 \pm 39$ & $115 \pm 44$ & $169 \pm 25$ & 0.008 & $88 \pm 14$ & $90 \pm 9$ & $178 \pm 113$ & $<0.001$ \\
\hline Colesterol total $(\mathrm{mg} / \mathrm{mL})$ & $194 \pm 46$ & $204 \pm 57$ & $221 \pm 89$ & 0.427 & $190 \pm 58$ & $176 \pm 44$ & $183 \pm 81$ & 0.439 \\
\hline $\mathrm{HDL}-\mathrm{C}(\mathrm{mg} / \mathrm{mL})$ & $32 \pm 8$ & $31 \pm 8$ & $30 \pm 5$ & 0.783 & $53 \pm 11$ & $50 \pm 9$ & $46 \pm 9$ & 0.254 \\
\hline LDL-C (mg/mL) & $112 \pm 35$ & $120 \pm 46$ & $118 \pm 24$ & 0.637 & $119 \pm 39$ & $115 \pm 35$ & $89 \pm 22$ & 0.300 \\
\hline Triglicéridos (mg/mL) & $137 \pm 90$ & $147 \pm 100$ & $344 \pm 280$ & $<0.001$ & $89 \pm 39$ & $140 \pm 94$ & $198 \pm 99$ & $<0.001$ \\
\hline
\end{tabular}

Número de individuos en paréntesis. *Test de ANOVA

Comparaciones múltiples (Test de Tukey)

Glucosa

Casos : TT vs. TC ( $p=0.511) ;$ TT vs. CC $(p=0.019) ;$ TC vs. CC $(p=0.006)$

Controles: TT vs. TC $(p=0.980) ;$ TT vs. CC $(p=0.001) ;$ TC vs. CC $(p<0.001)$

Triglicéridos

Casos : TT vs. TC ( $p=0.848) ;$ TT vs. CC $(p<0.001)$; TC vs. CC $(p<0.001)$

Controles : TT vs. TC $(p=0.039) ;$ TT vs. CC $(p<0.001) ;$ TC vs. CC $(p=0.039)$

\section{Discusión}

Diversas investigaciones han demostrado que las ECV poseen un importante componente genético ${ }^{31}$. Sin embargo, en nuestro país, aún son escasos los trabajos relacionados a este tema ${ }^{32-36}$. En el presente estudio fue evaluada, por primera vez en Chile, la frecuencia de los polimorfismos S19W y $-1131 \mathrm{~T}>\mathrm{C}$ del gen APOA5 y su posible contribución al desarrollo de EC en individuos de la IX Región de La Araucanía.

Al analizar las características bioquímicas de los individuos incluidos en este estudio, se encontraron concentraciones séricas de colesterol, LDL-C, triglicéridos, glucosa y ácido úrico significativamente más elevadas en el grupo casos al ser comparadas con el grupo control. Por otra parte, los valores de HDL-C fueron significativamente más bajos en los pacientes con enfermedad coronaria que en controles. Además, la presencia de factores de riesgo clásicos de EC fue significativamente mayor en los pacientes con EC.

El análisis molecular demostró que no existe asociación directa entre las variantes del gen APOA5 investigadas y enfermedad coronaria en los sujetos evaluados. La frecuencia de los alelos mutados fue similar entre casos y controles $(p>0.05)$. Resultados semejantes fueron observados por Martinelli et al. ${ }^{22}$ en población italiana, quienes no encontraron evidencias de asociación entre los polimorfismos S19W, $-1131 \mathrm{~T}>\mathrm{C}$ y la presencia de EC confirmada por angiografía en 669 sujetos. Por otro lado, diversos autores han relacionado estos polimorfismos con mayor susceptibilidad de desarrollar enfermedad coronaria en diferentes poblaciones ${ }^{18-21}$. Existen diversas razones que pueden explicar estas discrepancias, entre éstas podemos mencionar: el diseño del estudio, la definición de los criterios de inclusión y exclusión, el número de individuos, y por supuesto la etnia de los sujetos evaluados ${ }^{37}$.

Sin embargo, al evaluar la posible asociación entre los diferentes genotipos de las variantes investigadas y las concentraciones séricas de lípidos y glucosa en los individuos con enfermedad arterial coronaria y controles, se observó que los portadores de los alelos mutados 19W y -1131C presentaron niveles más elevados de triglicéridos y glucosa comparado a los portadores de los alelos normales. Similarmente, diversos autores han observado también estas asociaciones ${ }^{22,} 38,39$. Además, estas variantes genéticas han sido relacionadas con síndrome metabólico y diabetes mellitus tipo 2 ; todos factores de riesgo indiscutidos de enfermedad coronaria ${ }^{40,41}$.

En resumen, nuestro estudio muestra que los 


\section{N. Saavedra, A. Cuevas, A. Hernández, J. Caamaño, P. Jaramillo, F. Lanas, L. A. Salazar}

polimorfismos S19W y $-1131 \mathrm{~T}>\mathrm{C}$ del gen APOA5 no están asociados directamente a enfermedad coronaria en la población analizada. Sin embargo, estas variantes fueron relacionadas a niveles séricos más elevados de triglicéridos y glucosa, en ambos grupos; hallazgo que sugiere que estos polimorfismos pueden contribuir al origen de la dislipidemia diabética, un reconocido factor de riesgo de enfermedad arterial coronaria.

\section{REFERENCIAS:}

1. LANAS F. Factores de riesgo de infarto en América Latina. Estudio INTERHEARTH América latina. Rev Chil Cardiol 2007; 26:227-230.

2. FORRESTER JS. Triglycerides: risk factor or fellow traveler? Curr Opin Cardiol 2001; 16: 261-264

3. HOKANSON JE, AUSTIN MA. Plasma triglyceride level is a risk factor for cardiovascular disease independent of high-density lipoprotein cholesterol level: a meta-analysis of population-based prospective studies. J Cardiovasc Risk 1996; 3: 213-219

4. PENNACCHIO LA, OLIVIER M, HUBACEK JA, COHEN JC, COX DR, FRUCHART JC, et al. An apolipoprotein influencing triglycerides in humans and mice revealed by comparative sequencing. Science 2001; 294: 169-173

5. VAN DER VLIET HN, SAMMELS MG, LEEGWATER AC, LEVELS JH, REITSMA PH, BOERS W, et al. Apolipoprotein A-V: a novel apolipoprotein associated with an early phase of liver regeneration. J Biol Chem 2001; 276: 4451244520.

6. O'BRIEN PJ, ALBORN WE, SLOAN JH, ULMER $M$, BOODHOO A, KNIERMAN AD, et al. The novel apolipoprotein A5 is present in human serum, is associated with VLDL, HDL, and chylomicrons, and circulates at very low concentrations compared with other apolipoproteins. Clin Chem 2005; 51: 351-359.

7. VAN DER VLIET HN, SCHAAP FG, LEVELS JHM, OTTENHOFF R, LOOIJE N, WESSELING JG, et al. Adenoviral overexpression of apolipoprotein $A-V$ reduces serum levels of triglycerides and cholesterol in mice. Biochem Biophys Res Commun 2002; 295:1156-1159.

8. FRUCHART-NAJIB J, BAUGE E, NICULESCU LS, PHAM T, THOMAS B, ROMMENS C, et al. Mechanism of triglyceride lowering in mice expressing human apolipoprotein A5. Biochem Biophys Res Commun 2004; 319:397-404.

9. MERKEL M, HEEREN J. Give me A5 for lipoprotein hydrolysis!. J Clin Invest 2005; 115: 2694-6.

10. SUN G, BI N, LI G, ZHUX, ZENG X, WU G, etal. Identification of lipid binding and lipoprotein lipase activation domains of human apoAV. Chem Phys Lipids 2006; 143: 22-28.

11. TAIES, ORDOVAS JM. Clinical significance of apolipoprotein A5. Curr Opin Lipidol 2008; 19: 349-54.

12. KOMURCU-BAYRAK E, ONAT A, PODA M, HUMPHRIES SE, PALMEN J, GUCLU F, et al. Gender-modulated impact of apolipoprotein A5 gene (APOA5) - 1131T>C and c.56C>G polymorphisms on lipids, dyslipidemia and metabolic syndrome in Turkish adults. Clin Chem Lab Med 2008; 46: 778-84.
13. OLANO-MARTIN E, ABRAHAM EC, GILL-GARRISON R, VALDESAM, GRIMALDIK, TANG F, et al. Influence of apoA$\mathrm{V}$ gene variants on postprandial triglyceride metabolism: impact of gender. J Lipid Res 2008; 49: 945-53.

14. CHIEN KL, FANG WH, WEN HC, LIN HP, LIN YL, LIN SW, et al. APOA1/C3/A5 haplotype and risk of hypertriglyceridemia in Taiwanese. Clin Chim Acta 2008; 390: 56-62.

15. MORENO R, PEREZ-JIMENEZ F, MARIN C, MORENO JA, GÓMEZ P, BELLIDO $C$, et al. A single nucleotide polymorphism of the apolipoprotein A-V gene $-1131 \mathrm{~T}>\mathrm{C}$ modulates postprandial lipoprotein metabolism. Atherosclerosis 2006; 189: 163-8.

16. RENSEN PC, VAN DIJK KW, HAVEKES LM. Apolipoprotein $\mathrm{AV}$ : low concentration, high impact. Arterioscler Thromb Vasc Biol 2005; 25: 2445-7.

17. YAN SK, CHENG XQ, SONG YH, XIAO XH, BI N, CHEN BS. Apolipoprotein A5 gene polymorphism -1131T-->C: association with plasma lipids and type 2 diabetes mellitus with coronary heart disease in Chinese.

18. HSU LA, KO YL, CHANG CJ, HU CF, WU S, TENG MS, et al. Genetic variations of apolipoprotein A5 gene is associated with the risk of coronary artery disease among Chinese in Taiwan. Atherosclerosis 2006; 185: 143-9.

19. BI N, YAN SK, LI GP, YIN ZN, CHEN BS. A single nucleotide polymorphism -1131T>C in the apolipoprotein A5 gene is associated with an increased risk of coronary artery disease and alters triglyceride metabolism in Chinese. Mol Genet Metab 2004; 83: 280-6.

20. SZALAI C, KESZEI M, DUBA J, PROHÁSZKA Z, KOZMA GT, CSÁSZÁR A, et al. Polymorphism in the promoter region of the apolipoprotein $A 5$ gene is associated with an increased susceptibility for coronary artery disease. Atherosclerosis 2004; 173: 109-14.

21. VAESSEN SF, SCHAAP FG, KUIVENHOVEN JA, GROEN AK, HUTTEN BA, BOEKHOLDT SM, et al. Apolipoprotein $A-V$, triglycerides and risk of coronary artery disease: the prospective Epic-Norfolk Population Study. J Lipid Res 2006; 47: 2064-70.

22. MARTINELLI N, TRABETTI E, BASSIA, GIRELLI D, FRISO S, PIZZOLO F, et al. The $-1131 \mathrm{~T}>\mathrm{C}$ and S19W APOA5 gene polymorphisms are associated with high levels of triglycerides and apolipoprotein C-III, but not with coronary artery disease: an angiographic study. Atherosclerosis 2007; 191: 409-17.

23. FOSSATI P, MEDICCI R. Abstract Book. International symposium on cholesterol control and cardiovascular diseases: Prevention and therapy. Milan Italy, 1987. Apud: 
Bayer Corporation, Diagnostic Division, Tarrytown, N.Y., Cholesterol-Fast color.

24. FOSSATI P, PRENCIPE L. Serum triglycerides determined colorimetrically with an enzyme of low-density lipoprotein cholesterol in plasma without use of the preparative ultracentrifuge. Clin Chem 1982; 28: 2077-2080.

25.BURSTEIN M, SCHOLNICK HR, MORFIN R. Rapid method for the isolation of lipoproteins from human serum by precipitation with polyanions. J Lipid Res 1970; 11: 583595.

26. FRIEDEWALD WT, LEVY RI, FREDRICKSON DS. Estimation of the concentration of low-density lipoprotein cholesterol in plasma, without use of the preparative ultracentrifuge. Clin Chem 1972; 18: 499 - 502.

27. FOSSATI P, PRENCIPE L, BERTI G. Use of 3,5-dichloro2-hydroxybenzenesulfonic acid/4-aminophenazone chromogenic system in direct enzymic assay of uric acid in serum and urine. Clin Chem 1980; 26: 227-31.

28. BARHAM D, TRINDER P. An improved colour reagent for the determination of blood glucose by the oxidase system. Analyst 1972; 97(151): 142-145.

29. SALAZAR LA, HIRATA MH, CAVALLI SA, MACHADO MO, HIRATA RD. Optimized procedure for DNA isolation from fresh and cryopreserved clotted human blood useful in clinical molecular testing. Clin Chem 1998; 44: 1748-1750.

30. TALMUD PJ, HAWE E, MARTIN S, OLIVIER M, MILLER GJ, RUBIN EM, et al. Relative contribution of variation within the APOC3/A4/A5 gene cluster in determining plasma triglycerides. Hum Mol Genet. 2002; 11: 3039-46.

31. NAVARRO F. Bases genéticas de la enfermedad coronaria. Rev Esp Cardiol 2002; 55: 413-431.

32. JARAMILLO PC, MUÑOZ A, LANAS C, LANAS F, SALAZAR LA. Endothelial nitric oxide synthase G894T gene polymorphism in Chilean subjects with coronary artery disease and controls. Clin Chim Acta 2006; 371: 102-106.

33. JARAMILLO PC, LANAS C, LANAS F, SALAZAR LA. $786 \mathrm{~T}>\mathrm{C}$ polymorphism of the endothelial nitric oxide synthase gene in Chilean subjects with coronary artery disease and controls. Clin Chim Acta 2008; 387: 105-108.

34. HERRERA C, JARAMILLO P, LANAS F, SALAZAR LA. Polimorfismo 2212A $>G$ del gen PECAM-1 en individuos chilenos con enfermedad coronaria y controles. Rev Chil Cardiol 2008; 27: 147-152.

35. SALAZAR LA, ZAMBRANO T, JARAMILLO P, LANAS F. Frecuencia de la variante $23968 \mathrm{~A}>\mathrm{G}$ del gen PCSK9 en individuos chilenos con enfermedad coronaria confirmada por angiografía y controles. Rev Chil Cardiol 2008; 27: 4350.

36. CAAMAÑO J, JARAMILLO, LANAS C, LANAS F, SALAZAR LA. Factor XII 46C>T gene polymorphism in Chilean subjects with coronary artery disease and controls. Med Princ Pract 2009 (in press).

37.BACH J, ENDLER G, MANNHALTER C, HELLSTERN P. Coagulation factor XII (FXII) activity, activated factor XII, distribution of factor XII C46T gene polymorphism and coronary risk: reply to a rebuttal. J Thromb Haemost 2008; 6:1055-6.

38. SUCHANEK P, LORENZOVA A, POLEDNE R, HUBACEK JA. Changes of plasma lipids during weight reduction in females depends on APOA5 variants. Ann Nutr Metab 2008; 53(2):104-108.

39. CHARRIERE S, BERNARD S, AQALLAL M, MERLIN M, BILLON S, PERROT L, et al. Association of APOA5 $1131 \mathrm{~T}>\mathrm{C}$ and S19W gene polymorphisms with both mild hypertriglyceridemia and hyperchylomicronemia in type 2 diabetic patients. Clin Chim Acta 2008; 394(1-2):99-103.

40.DALLONGEVILLE J, COTTEL D, WAGNER A, DUCIMETIĖRE P, RUIDAVETS JB, ARVEILER D, et al. he APOA5 Trp19 allele is associated with metabolic syndrome via its association with plasma triglycerides. BMC Med Genet. 2008; 9:84.

41. BAUM L, NG MC, SO WY, POON E, WANG Y, LAM VK, et al. A case-control study of apoA5 -1131T-->C polymorphism that examines the role of triglyceride levels in diabetic nephropathy. J Diabetes Complications. 2007; 21:158-63. 